\title{
Using Multiple Hypotheses to Improve Depth-Maps for Multi-View Stereo
}

\author{
Neill D.F. Campbell ${ }^{1}$, George Vogiatzis ${ }^{2}$, \\ Carlos Hernández ${ }^{2}$, and Roberto Cipolla ${ }^{1}$ \\ 1 Department of Engineering, University of Cambridge, Cambridge, UK \\ ${ }^{2}$ Computer Vision Group, Toshiba Research Europe, Cambridge, UK
}

\begin{abstract}
We propose an algorithm to improve the quality of depth-maps used for Multi-View Stereo (MVS). Many existing MVS techniques make use of a two stage approach which estimates depth-maps from neighbouring images and then merges them to extract a final surface. Often the depth-maps used for the merging stage will contain outliers due to errors in the matching process. Traditional systems exploit redundancy in the image sequence (the surface is seen in many views), in order to make the final surface estimate robust to these outliers. In the case of sparse data sets there is often insufficient redundancy and thus performance degrades as the number of images decreases. In order to improve performance in these circumstances it is necessary to remove the outliers from the depth-maps. We identify the two main sources of outliers in a top performing algorithm: (1) spurious matches due to repeated texture and (2) matching failure due to occlusion, distortion and lack of texture. We propose two contributions to tackle these failure modes. Firstly, we store multiple depth hypotheses and use a spatial consistently constraint to extract the true depth. Secondly, we allow the algorithm to return an unknown state when the a true depth estimate cannot be found. By combining these in a discrete label MRF optimisation we are able to obtain high accuracy depthmaps with low numbers of outliers. We evaluate our algorithm in a multi-view stereo framework and find it to confer state-of-the-art performance with the leading techniques, in particular on the standard evaluation sparse data sets.
\end{abstract}

\section{Introduction}

The topic of multi-view stereo (MVS) reconstruction has become a growing area of interest in recent years with many differing techniques achieving a high degree of accuracy [1]. These techniques focus on producing watertight 3D models from a sequence of calibrated images of an object, where the intrinsic parameters and pose of the camera are known. In addition to providing a taxonomy of methods, [1] also provides a quantitative analysis of performance both in terms of accuracy and completeness. The top performers may be loosely divided into two groups. The first group make use of techniques such as correspondence estimation, local region growing and filtering to build up a final dense surface $[13,15,16]$. The second group make use of some form of global optimisation strategy on a volumetric representation to extract a surface $[5,14,6,12,7]$. A common strategy is to split the reconstruction process into two stages. The first is to estimate a series of depth-maps using local groups of the input images. The second stage then attempts to combine these into a global surface estimate, making use of registration and regularisation techniques. This two stage approach is an elegant formulation which allows different techniques to be chosen independently for the two stages. Some recent methods achieve a fast computation time by avoiding a global optimisation when merging depth-maps $[17,18]$. In this paper we focus on the first of the two stages - local depth-map estimation. 

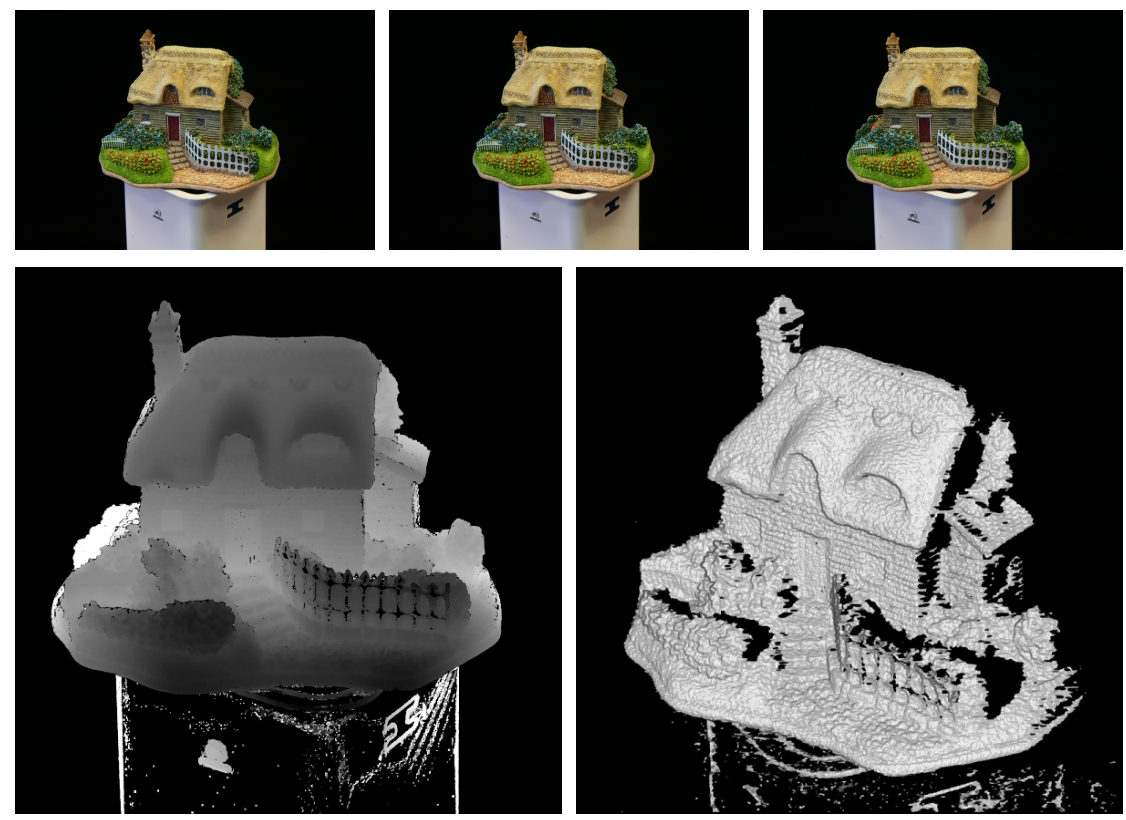

Fig. 1. Depth map obtained from only three images of a model house. The left image provides the recovered depth map which is rendered in the right image. As well as achieving a high degree of accuracy on surface detail our algorithm has correctly recovered the occlusion boundaries and removed outlying depth estimates

The estimation of local depth-maps is often performed using patch based methods [2]. The work of [5] proposed the use of Normalised Cross-Correlation (NCC) as the matching cost between two patches. This method offers good performance for textured objects and has been the basis of $[7,6,19]$. In the first stage of [5] a depth is estimated for each pixel independently. In the next stage the algorithm looks for consensus in depth estimates from multiple depth-maps. Since the individual depth-maps are known to contain outliers, this stage relies upon redundancy in the depth-maps to reject the them. In data-sets containing a large number of images (50-100) this approach performs quite well. In so called sparse data-sets (10-20 images) one expects very little redundancy in the reconstructed depth-maps, leading to a drop in reconstruction accuracy. This drop is actually observed in the performance of [5] in sparse data-sets with ground truth [1].

In this paper we show that if individual depth-maps are filtered for outliers prior to the fusion stage, good performance can be maintained in sparse data-sets. Our strategy is to collect a list of good hypotheses for the depth of each pixel. We then chose the optimal depth for each pixel by enforcing consistency between neighbouring pixels in a depth-map. A crucial element of the filtering stage is the introduction of a possible unknown depth hypothesis for each pixel, which is selected by the algorithm when no consistent depth can be chosen. This pre-processing of the depth-maps allows the global fusion stage to operate on fewer outliers and consequently improve the performance under sparsity of data.

The rest of the paper is laid out as follows: In $\S 2$ we review relevant prior work and discuss the differences of our approach. $\S 3$ presents the use of NCC as a photo-consistency metric for estimating depth-maps and provides an overview of our algorithm to reduce outliers. $\S 4$ provides the details of our depth-map estimation algorithm, in particular the optimisation process. In $\S 5$ we show how to extend an existing MVS framework to include our depth-map estimation 
procedure for the purpose of the experimental evaluation provided in $\S 6$. Here we display the improvements made to estimated depth-maps and also provided a quantitative evaluation of the MVS results. The paper concludes with our findings in $\S 7$. This work was supported by a Schiff Scholarship and Toshiba Research Europe.

\section{Previous Work}

A taxonomy of the established methods for dense stereo may be found in [2]. Most of these methods use matching costs to assign each pixel to a set of disparity levels within the image. The earlier algorithms maintained relatively few separate levels and were more targeted towards depth based segmentation rather than detailed reconstruction. The latest algorithms [3] obtain depth-maps with greater accuracy. Since these algorithms only have pairs of images available, they can make no use of redundancy across multiple images in a data set and thus they use spatial regularisation and optimisation schemes which attempt to infer information about the depths. Whilst we also exploit a spatial regularisation constraint, we only allow the optimisation to choose from a set of discrete depths, well localised by the NCC peaks. This contrasts with methods which allow the depth of each pixel to vary continuously whilst minimising some cost function.

Some of the best performing algorithms make use of an occluded state. This may be via an explicit estimation of a disparity map, for example [20] or internally as part of an optimisation routine [4]. We make use of the unknown state in a similar manner however we also use it recognise the other failure modes of NCC matching, discussed in $\S 3$, since they are indistinguishable.

The work of [5] proposed the robust NCC matching technique which we extend in our algorithm. Outlier rejection is accomplished through redundancy in the image sequence. The works of $[7,6]$ have used derivatives of this technique with slight modifications, for example the inclusion of a Parzen window to filter the consensus matches in [6]. The work of [19] proposed a new, color normalised supersampling approach to correct for projective warping errors and also provided improved computation time with an efficient GPU implementation.

Recent work has demonstrated that depth-map estimation and integration paradigm may be used to produce accurate results with greatly reduced computation time [18] or real-time [17]. Again the reliance upon redundancy in the image sequence is paramount, for example the visibility computations of [17].

Since our contribution affects only the depth-map estimation, the global stage may be considered separately. The works of $[23,24]$ present complementary algorithms for range image integration. Here, the depth-maps produced by our algorithm would provide a suitable set of range images. The use of a volumetric graph-cut to extract the surface was proposed in [14] and extended in [6] to include the robust NCC photoconsistency. Other works have shown the graph-cut formulation to perform well as a global optimisation stage [12, 21].

The work of [22] uses multiple depth hypotheses as a result of reflections during the active 3D scanning of specular objects. Here a different framework, also based on spatial consistency, is used to reject false matches. The work of [26] makes use of multiple hypotheses for the related problem of new-view synthesis. They also make use of an MRF optimisation, here using a truncated quadratic kernel, to solve their synthesis problem. 


\section{Normalised Cross-Correlation for Photo-Consistency}

Normalised Cross Correlation (NCC) may be used to define an error metric for matching two windows in different images. Figure 2 provides an example of using NCC and epipolar geometry to perform window based matching. If we fix a pixel location in a reference image, for each possible depth away from that pixel we get a corresponding pixel in the second image. By computing the NCC between windows centred in those two pixels we can define a matching score as a function of depth for the reference pixel. We refer to this function as the correlation curve of the pixel. A typical correlation curve will exhibit a very sharp peak at the correct depth, and possibly a number of secondary peaks in other depths.

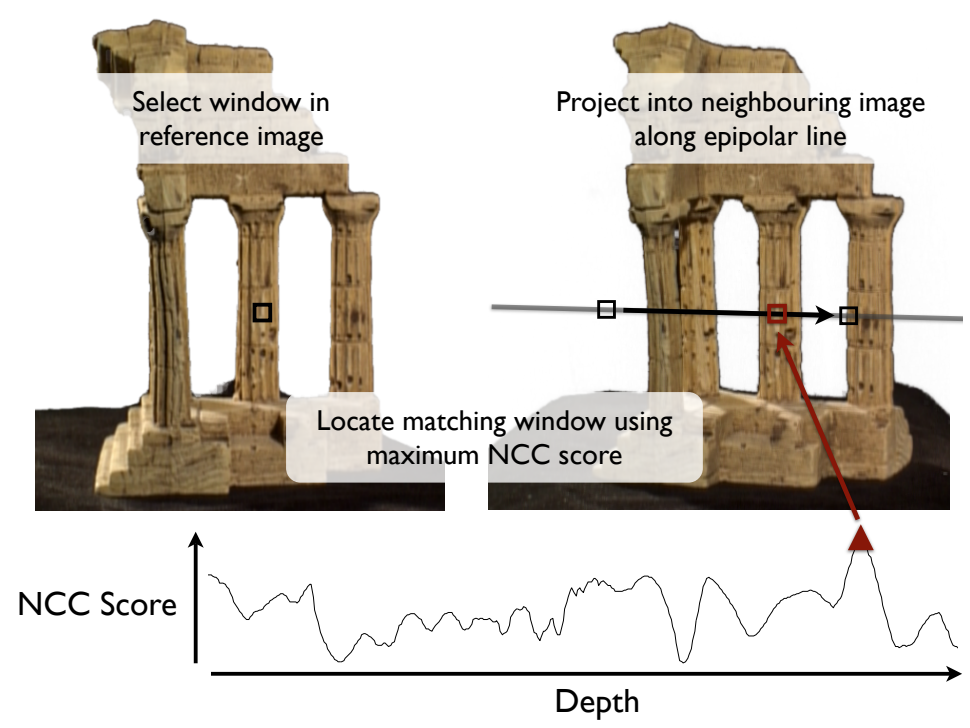

Fig. 2. Normalised Cross-Correlation based window matching.

In [5] a depth-map is generated for each input image using this matching technique for neighbouring images. For each pixel a number of correlation curves are computed (using a few of the neighbouring viewpoints) and the depth that gives rise to most peaks in those curves is selected as the depth for that pixel. See [5] or [6] for details. This process results in an independent depth estimate for each pixel. These depth estimates will unavoidably contain a significant percentage of outliers which must be dealt with in the subsequent step of [5] which is the volumetric fusion of multiple depth-maps. In data sets with a large number of images this is is overcome by the redundancy in the depth-estimates. The same surface point is expected to be covered by many different depth-maps, some of which will have the right depth estimate. In sparse data-sets however, each surface point may be seen by as few as two or three depth-maps. It is therefore crucial that outliers are minimised in the depth-map generation stage.

In this work we focus on the two most significant failure modes of NCC matching which are (1) the presence of repetitions in the texture and (2) complete matching failure due to occlusion, distortion and lack of texture. These are now described in more detail. 


\subsection{Repeating texture}

In general, there is no guarantee that the appearance of a patch is unique across the surface of the object. This results in correlation curve peaks at incorrect depths due to repeated texture - 'false' matches (Fig. 2). A larger window size is more likely to uniquely match to the true surface, reducing the number of false matches. However the associated peak will be broader and less well localised, reducing the accuracy of the depth estimate. The absolute value of the NCC score at a peak reflects how well the two windows match. Thus one might expect the peak with the maximum score to be the true peak. Unfortunately, the appearance of false matches due to repeated texture may result in false peaks having similar or even greater scores than the true surface peak (Fig. 3 (a)). To identify the correct peak, we propose to apply a spatial consistency constraint across neighbouring pixels in the depth-map. The underlying assumption is that if a peak corresponds to the true surface, the neighbouring pixels should have peaks at a similar depth. The exception to this is occlusion boundaries, which are however catered for under the next failure mode.

\subsection{Matching failure}

The second failure mode is comprised of occlusion errors, distorted image windows (due to slanted surfaces) and lack of texture. In all of these cases, the correlation curve will not exhibit a peak at the true depth of the surface, resulting in only false peaks. Furthermore no spatial consistency can be enforced between the pixel in question and its neighbours. In this situation we would like to acknowledge that the depth at this pixel is unknown and should therefore offer no vote for the surface location.

In order to achieve these two goals we propose an optimisation strategy which makes use of a discrete label Markov Random Field (MRF). The MRF allows each pixel to choose a depth corresponding to one of the top NCC peaks which is spatially consistent with neighbouring pixels or select an unknown label to indicate that no such peak occurs and there is no correct depth estimate. This process means that the returned depth map should only contain accurate depths, estimated with a high degree of certainty, and an unknown label for pixels which have no certain associated depth. Figure 3 illustrates the optimisation for a 1D example of neighbouring pixels across an occlusion boundary.

\section{Depth Map Estimation}

Our proposed algorithm estimates the depth for each pixel in the input images. It proceeds in two stages: Initially we extract a set of possible depth values for each pixel using NCC as a matching metric. We then solve a multi-label discrete MRF model which yields the depth assignment for every pixel. One of the key features in this process is the inclusion of an unknown state in the MRF model. This state is selected when there is insufficient evidence for the correct depth to be found. 


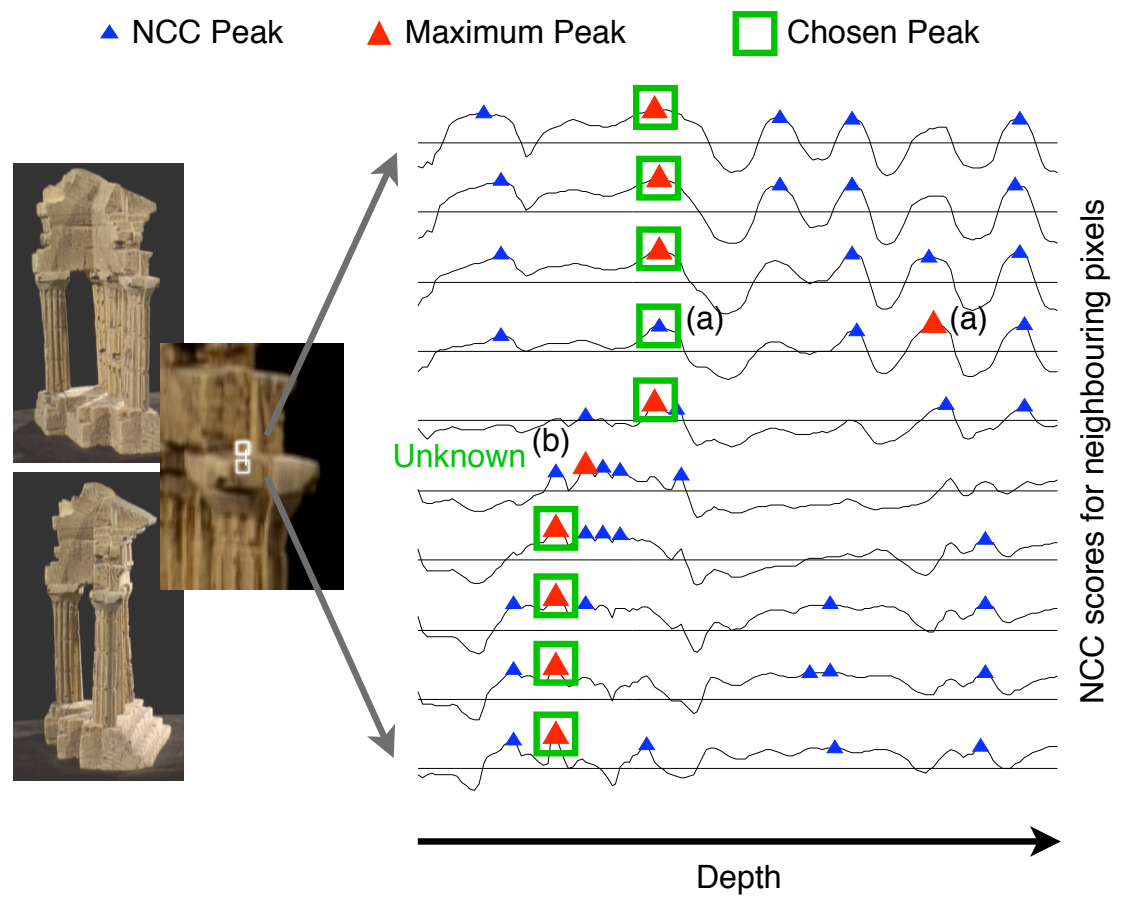

Fig. 3. Illustration of the MRF optimisation applied to neighbouring pixels. Existing method return the maximum peak which results in outliers in the depth estimate. The MRF optimisation corrects an outlier to the true surface peak (a) and introduces an unknown label at the occlusion boundary (b)

\subsection{Candidate Depths}

The input to our algorithm is a set of calibrated images $\mathcal{I}$ and the output is a set of corresponding depth-maps $\mathcal{D}$. In the following, we describe how to acquire a depth-map for a reference image $I_{\text {ref }} \in \mathcal{I}$. Let $N\left(I_{\text {ref }}\right)$ denote a set of 'neighbouring' images to $I_{\text {ref }}$.

As proposed in $\S 3$, we wish to obtain a hypothesis set of possible depths for each pixel $p_{i} \in I_{\text {ref }}$. Taking each pixel in turn, we project the epipolar ray into a second image $I_{n} \in I_{\text {ref }}$ and sample the NCC matching score over a depth range $\rho_{i}(z)$. We compute the score using a rectangular window centred at the projected image co-ordinates. One of the advantages of the multiple depth hypotheses is the ability to use a smaller matching window to provide a faster computation and improved localisation of the surface. Once we have obtained the sampled ray we store the top $K$ peaks $\hat{\rho}_{i}\left(z_{i, k}\right), k \in[1, K]$ with the greatest NCC score for each pixel. Depending on the number of images available, and the width of the camera baseline, this process may be repeated for other neighbouring images. We then continue to the optimisation stage with a set of the best $K$ possible depths, and their corresponding NCC scores, over all neighbouring images of $I_{\text {ref }}$.

\subsection{MRF Formulation}

At this stage a set of candidate depths $\hat{\rho}_{i}\left(z_{i, k}\right), k \in[1, K]$, for each pixel $p_{i}$ in the reference image $I_{\text {ref }}$ has been assigned and we wish to determine the correct depth map label for each pixel. As described in $\S 3$, we also make use of an unknown state to account for the failure modes of NCC matching. 
We model the problem as a discrete MRF where each each pixel has a set of up to $(K+1)$ labels. The first $K$ labels, fewer if an insufficient number of peaks were found during the matching stage, correspond to the peaks in the NCC function and have associated depths $z_{i, k} \in \mathcal{Z}_{i}$ and scores $\hat{\rho}_{i}\left(z_{i, k}\right)$. The final state is the unknown state $\mathcal{U}$. If the optimisation returns this state, the pixel is not assigned a depth in the final depth map. For each pixel we therefore form an augmented label set $z_{i, k}^{\prime} \in\left\{\mathcal{Z}_{i}, \mathcal{U}\right\}$ to include the unknown state.

The optimisation assigns a label $\left.\bar{k}_{i} \in\{1 \ldots K, \mathcal{U})\right\}$ to each pixel $p_{i}$. The cost function to be minimised consists of unary potentials for each pixel and pairwise interactions over first order cliques. The cost of a labelling $\overline{\mathbf{k}}=\left\{\bar{k}_{i}\right\}$ is expressed as

$$
E(\overline{\mathbf{k}})=\sum_{i} \phi\left(\bar{k}_{i}\right)+\sum_{(i, j)} \psi\left(\bar{k}_{i}, \bar{k}_{j}\right)
$$

where $i$ denotes a pixel and $(i, j)$ denote neighbouring pixels.

The following sections discuss the formulation of the unary potentials $\phi(\cdot)$ and pairwise interactions $\psi(\cdot, \cdot)$.

\subsection{Unary Potentials}

The unary labelling cost is derived from the NCC score of the peak. We wish to penalise peaks with a lower matching score since they are more likely to correspond to an incorrect match due to occlusion or noise. The NCC process will always return a score in the range $[-1,1]$. As is common practice, [6], we take an inverse exponential function to map this score to a positive cost.

The unary cost for the unknown state is set to a constant value $\phi_{\mathrm{U}}$. This term serves two purposes. Firstly it acts as a cut-off threshold for peaks with poor NCC scores which have no pairwise support (neighbouring peaks of similar depth). This mostly accounts for peaks which are weakly matched due to distortion or noise. Secondly it acts as a truncation on the depth disparity cost of the pairwise term. By assigning a low pairwise cost between peaks and the unknown state, the constant unary cost will effectively act as a threshold on the depth disparity to handle the case of an occlusion boundary. Thus the final unary term is given by

$$
\phi\left(k_{i}=x\right)=\left\{\begin{array}{ll}
\lambda e^{-\beta \hat{\rho}_{i}\left(z_{i, x}\right)} & x \in[1 \ldots K] \\
\phi_{\mathrm{U}} & x=\mathcal{U}
\end{array} .\right.
$$

\subsection{Pairwise Interactions}

The pairwise labelling cost is derived from the disparity in depths of neighbouring peaks. As has been previously mentioned, this term is not intended to provide a strong regularisation of the depth map. Instead it is used to try and determine the correct peak, corresponding to the true surface location, out of the returned peaks. We observe that the correct peak may not have the maximum score. Therefore if there is strong agreement on depth between neighbouring peaks, we take this to be the true location of the surface.

When dealing with the depth disparity term we are really considering surface orientation; whether the surface normal is pointing towards or away from the camera. Under a perspective 
projection camera model it is therefore necessary to correct for the absolute depth of the peaks rather than simply taking the difference in depth. We perform this correction by dividing by the average depth of the two peaks. The resulting pairwise term is given by

$$
\psi\left(k_{i}=x, k_{j}=y\right)=\left\{\begin{array}{lll}
2 \frac{\left|z_{i, x}-z_{j, y}\right|}{\left(z_{i, x}+z_{j, y}\right)} & x \in[1 \ldots K] & y \in[1 \ldots K] \\
\psi_{\mathrm{U}} & x=\mathcal{U} & y \in[1 \ldots K] \\
\psi_{\mathrm{U}} & x \in[1 \ldots K] & y=\mathcal{U} \\
0 & x=\mathcal{U} & y=\mathcal{U}
\end{array} .\right.
$$

We set $\psi_{\mathrm{U}}$ to a small value to encourage regions with many pixels labelled as unknown to coalesce. This acts as a further stage of noise reduction since it prevents spurious peaks with high scores but no surrounding support from appearing in regions of occlusion.

\subsection{Optimisation}

To obtain the final depth map we need to determine the optimal labelling $\hat{\mathbf{k}}$ such that

$$
E(\hat{\mathbf{k}})=\arg \min _{(\overline{\mathbf{k}})} \sum_{i} \phi\left(\bar{k}_{i}\right)+\sum_{(i, j)} \psi\left(\bar{k}_{i}, \bar{k}_{j}\right)
$$

Since in the general case this is an NP-hard problem we must use an approximate minimisation algorithm to achieve a solution. The most well-known techniques for solving problems of this nature are based on graph-cuts and belief propagation. Instead, we use the recently developed sequential tree-reweighted message passing algorithm, termed TRW-S, of [8]. This has been shown to outperform belief propagation and graph-cuts in tests on stereo matching using a discrete number of disparity levels. In addition to minimising the energy, the algorithm estimates a lower bound on the energy at each iteration which is useful in checking for convergence and evaluating the performance of the algorithm. We should note, however, that we are by no means guaranteed that the lower bound is attainable.

\section{Extension to Multi-View Stereo Framework}

As previously discussed, the detailed evaluation of [1] demonstrates that volumetric methods display state-of-the-art performance both in terms of accuracy and completeness. Some of the most successful create a 3D cost field within a volume and the reconstruction task is then to extract the optimal surface from this volume. Algorithms developed for segmentation problems are commonly used to extract the surface.

In order to evaluate the improvement to multi-view stereo we combined our depth map estimation with a modified implementation of the volumetric regularisation framework of [6]. This method uses a volumetric graph-cut to recover the surface from an array of voxels. Each voxel becomes a node in a 3D binary MRF where the voxel must be labelled as inside or outside the object. The MRF formulation allows for two terms in the cost function. The first is the unary foreground/background labelling cost. This encodes the likelihood that a particular voxel is part of the object or empty space. The recent work of [9] shows how depth maps may be used 
to evaluate a probabilistic visibility measure for each voxel in the volume. This term may be used to estimate whether or not the voxel in question resides in empty space and is therefore visible from the cameras. From this it is possible to derive an appropriate cost for the unary term related to the likelihood of visibility. The second term is the pairwise discontinuity cost. This term represents the likelihood that the surface boundary lies between two neighbouring voxels. This term may be derived directly from the individual depth maps projected into the volume.

In [25] the authors show that the energy cost is a discrete approximation to the sum of a weighted surface area of the boundary (the pairwise terms) and a weighted volume of the object (the unary terms). This framework is ideal for use with our depth maps since it provides global regularisation using all the available data. This is a key advantage of our approach. Rather than perform regularisation on individual depth maps to recover uncertain regions, we only return depths with a high degree of confidence associated with them. Thus other depth maps may be able to fill in the areas where a particular depth map is uncertain. In the event that there are still regions of the surface which are not determined precisely by any of the depth maps, the regularisation should be performed by a global method which takes into account the data from all the depth maps rather than an amalgamation of estimates from individual depth maps.

\subsection{Depth Map Acquisition}

The first stage of the reconstruction process is to acquire the depths maps. Our method is to select an image and project rays into the nearest neighbouring images in a sequential process. We maintain a cumulative store of the $K$ top scoring NCC peaks for each pixel. This provides an even greater degree of robustness against occlusion than the technique of [6] and is easier to implement in a parallel environment such as a GPU. Rather than requiring peaks from multiple images to fall in the same location, we only have to accurately observe a surface location in a single pair of images and rely on the surrounding support of peaks to identify the correct peaks. The speed of the depth map computation maybe increased by using the object silhouettes to avoid performing NCC matching calculations in regions outside the possible surface locations. Extraction of silhouettes for multi-view stereo may be performed as an automatic process [10].

\subsection{Surface Recovery}

Integrating our depth maps with the framework of [6] and [9] is a simple and elegant process. For the visibility volume we may project the same probability of visibility along each ray as [9] when we have a known depth. For pixels labelled as unknown we simply project a likelihood of 0.5 to indicate that this pixel provides no information about visibility. For the discontinuity cost we adopt a 'binning' approach. For each voxel in the discontinuity volume we take the sum of the projected depths of all the pixels in all the depth maps which fall inside the voxel, weighted by their NCC scores. If a pixel is labelled as unkown then it plays no part in the discontinuity cost. The final optimisation follows in the same manner as [6] with the graph-cut used to segment the volume. The iso-surface is extracted and smoothed using a snake [5] to perform 'intelligent' smoothing making use of the photoconsistency volume. 


\section{Experiments}

\subsection{Implementation}

To improve the computation time for our depth maps we perform the NCC matching by taking advantage of the parallel processing and texture facilities of the GPU of modern graphics cards. The GPU code improves performance by up to an order of magnitude depending on the window size. On of the advantages of our method is the ability to use small windows which result in greater precision of the surface location but which introduce a significant amount of noise which will adversely affect many of the existing techniques. The use of the smaller window also results in a greater saving in computational efficiency since the GPU offers improved performance with small kernels.

For the optimisation of the discrete MRF for the depth map we use the TRW-S implementation of Kolmogorov [8]. We also use Kolmogorov's implementation of the graph-cut algorithm [11]. Our implementation, running on a $3.0 \mathrm{GHz}$ machine with an nVidia Quadro graphics card, can evaluate $900 \mathrm{NCC}$ depth slices in 20 seconds for the temple sequence (image resolution $640 \times 480)$. The TRW-S optimisation has a typical run time of 20 seconds for the same images. The final volumetric graph-cut typically runs in under 5 minutes for a $350^{3}$ voxel array.

For all the experiments we used the following parameter values: $\beta=1, \lambda=1, \phi_{\mathrm{U}}=0.04$ and $\psi_{\mathrm{U}}=0.002$. We used an NCC window size of $5 \times 5$.

\subsection{Depth Maps}

Fig. 4 illustrates the improvement of our method over the voting schemes of $[5,6]$. Fig. 4 (b) shows the depth that would be determined by simply taking the NCC peak with the greatest score. Our method, implemented here with $K=9$ peaks, is able to select the peak corresponding to true surface peak from the ranked candidate peaks and Fig. 4 (d) illustrates that a significant proportion of the true surface peaks are not the absolute maximum. We also observe that pixels are correctly labelled with the unknown state along occlusion boundaries and along areas such as the back wall of the temple and edges of the pillars where the surface normal is oriented away from the camera. Looking at the rendering of this depth-map and its neighbour, Fig. 4(e-g), we can observe that very few erroneous depths are recovered and we observe that the combination of the two depths maps align and complement each other rather than attempting to fill in the holes on the individual depth-maps which would impact the subsequent multi-view stereo global optimisation.

Fig. 5 shows the results on the 'cones' dataset which forms part of the standard dense stereo evaluations images and consists of a single stereo pair with the left image shown. Our depthmap again shows a high degree of detail on textured surfaces and we correctly identify occlusion boundaries with the unknown state. Further more the algorithm also correctly textures the failure modes of NCC by returning the unknown state in texture-less regions where the matching fails to accurately localise the surface.

\subsection{Multi-View Stereo Evaluation}

In order to evaluate the improvement of our depth-maps to multi-view stereo we ran our algorithm on the standard evaluation 'temple' dataset. The following table provides the accuracy 
(a)

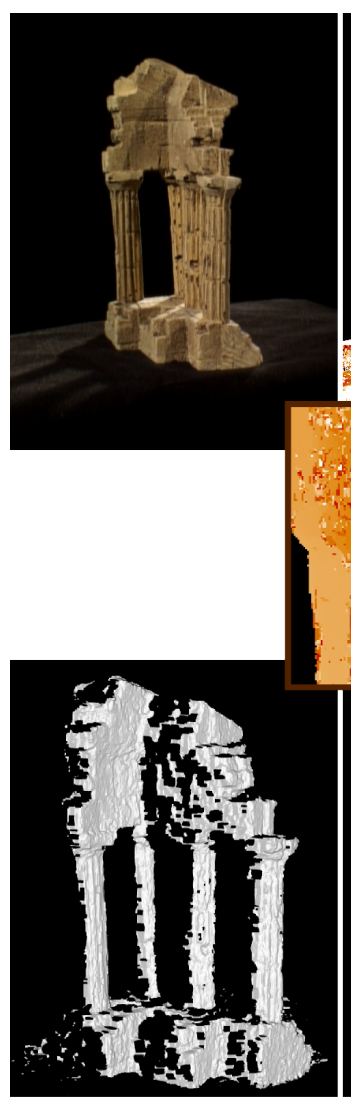

(e) (b)

(c)

(d)

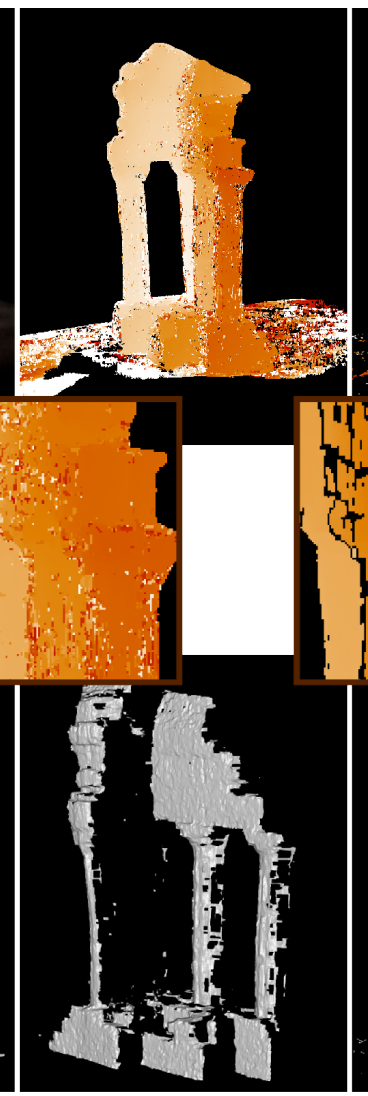

(f)

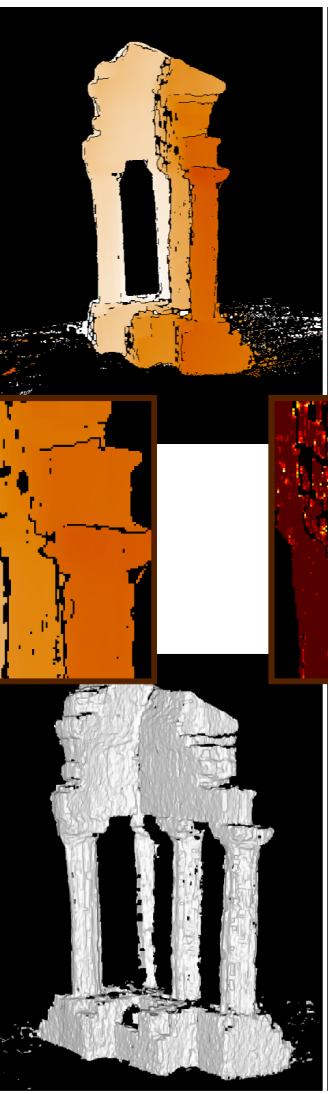

(g)

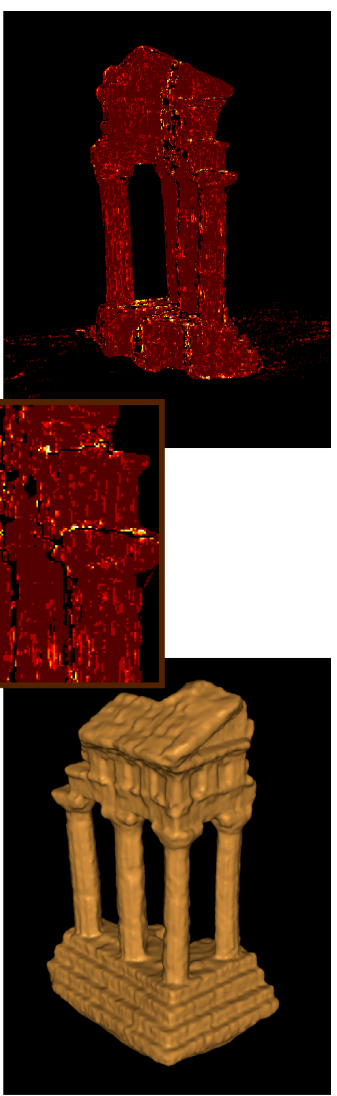

(h)

Fig. 4. Results of the depth map estimation algorithm. Two neighbouring images are combined with the reference image (a). If we simply took the NCC peak with the maximum score, as in [5], we would obtain (b). The result of our algorithm (c) shows a significant reduction in noise. We have corrected noisy estimates of the surface and the unknown state has also been used to clearly denote occlusion boundaries and remove poorly matched regions. The number of the correct surface peak returned, ranked by NCC score, is displayed in (d) where dark red indicates the peak with the greatest score. The rendered depth-map is shown in (e) along with the neighbouring depth-map (f) with (g) showing the two superimposed. The final reconstruction (h) for the sparse temple sequence (16 images) of [1]

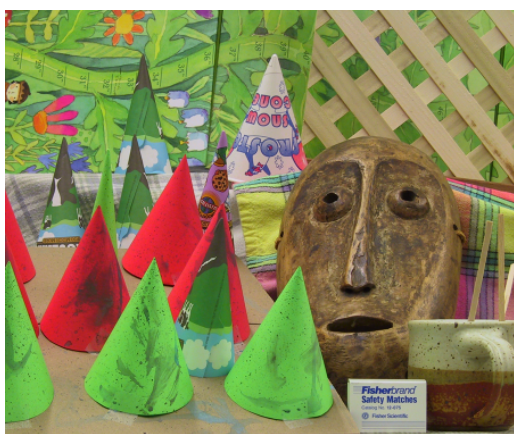

(a)

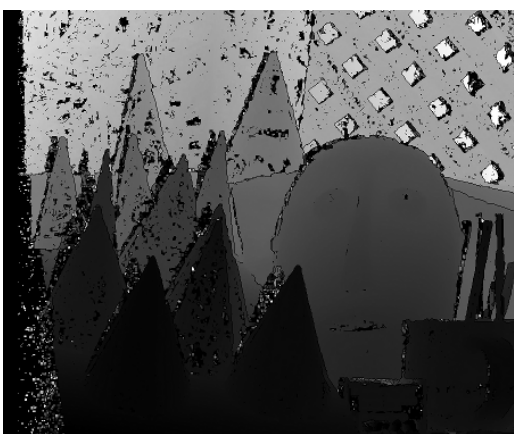

(b)

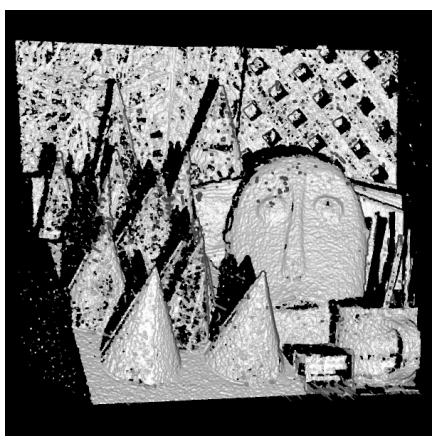

(c)

Fig. 5. Single view stereo results for the 'Cones' data set. The left image of the stereo pair is shown in (a) with the recovered depth-map in (b), rendered in (c) 
and completeness measures of [1] against the ground-truth data for the object. In terms of both accuracy and completeness our results provide a significant improvement in both the sparse ring and ring datasets. In particular we observe that the results for the sparse ring offer greater accuracy than the other algorithms [3] running on the ring sequence (3 times as many images) with the exception of [5].

\begin{tabular}{l|c|c|c} 
& \multicolumn{3}{|c}{ Accuracy / Completeness } \\
& Full (312 images) & Ring (47 images) & SparseRing (16 images) \\
\hline Our Results & $\mathbf{0 . 4 1} \mathbf{m m ~ / ~ 9 9 . 9 \%}$ & $\mathbf{0 . 4 8 m m ~ / ~ 9 9 . 4 \% ~}$ & $\mathbf{0 . 5 3 m m ~ / ~ 9 8 . 6 \%}$ \\
\hline
\end{tabular}

\section{Conclusions}

The results of our experiments confirm that our method offers a significant improvement in performance over the current state-of-the-art reconstruction algorithms when running on sparse data sets. By explicitly accounting for the failure modes of the NCC matching technique we are able to produce depth-maps which accurately locate the true surface in noise, allowing the use of small matching windows. We are also able to identify when the surface estimate is inconsistent, due to lack of texture or occlusion, and label pixels as having unknown depths. Returning this unknown state, rather than providing a form of local regularisation, allows a subsequent global regularisation to be performed over all the depth-maps using the best possible data. If there are unknown surface regions which are not recovered by the depth-map a global regularisation scheme is in a much better position to estimate the surface since it has access to all of the depth-maps. This is particularly true in the case of the sparse ring temple dataset and we believe is primarily responsible for its improved performance over other methods. We also note that our depth-map estimation algorithm may be integrated with a variety of multi-view stereo algorithms $[5-7,12,17,18,21]$ where is should confer similar increases in performance.

\section{References}

1. Seitz, S., Curless, B., Diebel, J., Scharstein, D., Szeliski, R.: A comparison and evaluation of multi-view stereo reconstruction algorithms. In: Proc. IEEE Conf. on Computer Vision and Pattern Recognition. (2006)

2. Scharstein, D., Szeliski, R.: A taxonomy and evaluation of dense two-frame stereo correspondence algorithms. Intl. Journal of Computer Vision 47(1-3) (2002)

3. http://vision.middlebury.edu/

4. Criminisi, A., Shotton, J., Blake, A., Rother, C., Torr, P.: Efficient dense stereo with occlusions for new view-synthesis by four-state dynamic programming. Intl. Journal of Computer Vision 71(1) (2007)

5. Hernández, C., Schmitt, F.: Silhouette and stereo fusion for 3d object modeling. Computer Vision and Image Understanding 96(3) (December 2004)

6. Vogiatzis, G., Hernández, C., Torr, P.H.S., Cipolla., R.: Multi-view stereo via volumetric graph-cuts and occlusion robust photo-consistency. IEEE Trans. Pattern Anal. Mach. Intell. 29(12) (2007)

7. Goesele, M., Curless, B., Seitz, S.: Multi-view stereo revisited. In: Proc. IEEE Conf. on Computer Vision and Pattern Recognition. (2006)

8. Kolmogorov, V.: Convergent tree-reweighted message passing for energy minimization. IEEE Trans. Pattern Anal. Mach. Intell. 28(10) (2006)

9. Hernández, C., Vogiatzis, G., Cipolla, R.: Probabilistic visibility for multi-view stereo. In: Proc. IEEE Conf. on Computer Vision and Pattern Recognition. (2007)

10. Campbell, N.D.F., Vogiatzis, G., Hernández, C., Cipolla, R.: Automatic 3d object segmentation in multiple views using volumetric graph-cuts. In: 18th British Machine Vision Conference. Volume 1. (2007) 
11. Boykov, Y., Kolmogorov, V.: An experimental comparison of min-cut/max-flow algorithms for energy minimization in vision. IEEE Trans. Pattern Anal. Mach. Intell. 26(9) (September 2004)

12. Hornung, A., Kobbelt, L.: Hierarchical volumetric multi-view stereo reconstruction of manifold surfaces based on dual graph embedding. In: Proc. IEEE Conf. on Computer Vision and Pattern Recognition. (2006)

13. Furukawa, Y., Pons, J.: Accurate, dense, and robust multi-view stereopsis. In: Proc. IEEE Conf. on Computer Vision and Pattern Recognition. (2007)

14. Vogiatzis, G., Torr, P., Cipolla, R.: Multi-view stereo via volumetric graph-cuts. In: Proc. IEEE Conf. on Computer Vision and Pattern Recognition. (2005)

15. Habbecke, M., Kobbelt, L.: A surface-growing approach to multi-view stereo reconstruction. In: Proc. IEEE Conf. on Computer Vision and Pattern Recognition. (2007)

16. Goesele, M., Snavely, N., Curless, B., Hoppe, H., Seitz, S.: Multi-view stereo for community photo collections. In: Proc. $11^{\text {th }}$ Intl. Conf. on Computer Vision. (2007)

17. Merrell, P., Akbarzadeh, A., Wang, L., Mordohai, P., Frahm, J.-M., Yang, R., Nistér, D., Pollefeys, M.: Real-time visibility-based fusion of depth maps. In: Proc. $11^{\text {th }}$ Intl. Conf. on Computer Vision. (2007)

18. Bradley, D., Boubekeur, T., Heidrich, W.: Accurate multi-view reconstruction using robust binocular stereo and surface meshing. In: Proc. IEEE Conf. on Computer Vision and Pattern Recognition. (2008)

19. Hornung, A., Kobbelt, L.: Robust and efficient photoconsistency estimation for volumetric 3D reconstruction. In: Proc. $9^{\text {th }}$ Europ. Conf. on Computer Vision. (2006)

20. Sun, J., Li, Y., Kang, S.B., Shum, H.-Y.: Symmetric stereo matching for occlusion handling. In: Proc. IEEE Conf. on Computer Vision and Pattern Recognition. (2005)

21. Sinha, S.N., Mordohai, P., Pollefeys, M.: Multi-view stereo via graph cuts on the dual of an adaptive tetrahedral mesh. In: Proc. $11^{\text {th }}$ Intl. Conf. on Computer Vision. (2007)

22. Park, J., Kak, A.C.: Multi-peak range imaging for accurate 3D reconstruction of specular objects. In: Proc $6^{\text {th }}$ Asian Conf. on Computer Vision. (2004)

23. Curless, B., Levoy, M.: A volumetric method for building complex models from range images. In: Proc. of the ACM SIGGRAPH '96. (1996)

24. Zach, C., Pock, T., Bischof, H.: A globally optimal algorithm for robust TV-L1 range image integration. In: Proc. $11^{\text {th }}$ Intl. Conf. on Computer Vision. (2007)

25. Boykov, Y., Kolmogorov, V.: Computing geodesics and minimal surfaces via graph cuts. In: Proc. $9^{\text {th }}$ Intl. Conf. on Computer Vision. (2003)

26. Woodford, O.J., Reid, I.D., Fitzgibbon, A.W.: Efficient new view syntesis using pairwise dictionary priors. In: Proc. IEEE Conf. on Computer Vision and Pattern Recognition. (2007) 